\title{
Chemical Agent Incident-Response and Recovery Decision Process
}

\section{August 24, 2009}

\section{Primary Authors:}

Ellen Raber, Robert Greenwalt, Wilthea Hibbard, Don MacQueen, Sav Mancieri, and Dennis Reutter

Lawrence Livermore National Laboratory (LLNL)

With contributions from:

Mark D. Tucker, Donna M. Edwards, Robert Knowlton, Rita Betty, and Paula Krauter

Sandia National Laboratories (SNL)



\section{DISCLAIMER}

This document was prepared as an account of work sponsored by an agency of the United States government. Neither the United States government nor Lawrence Livermore National Security, LLC, nor any of their employees makes any warranty, expressed or implied, or assumes any legal liability or responsibility for the accuracy, completeness, or usefulness of any information, apparatus, product, or process disclosed, or represents that its use would not infringe privately owned rights. Reference herein to any specific commercial product, process, or service by trade name, trademark, manufacturer, or otherwise does not necessarily constitute or imply its endorsement, recommendation, or favoring by the United States government or Lawrence Livermore National Security, LLC. The views and opinions of authors expressed herein do not necessarily state or reflect those of the United States government or Lawrence Livermore National Security, LLC, and shall not be used for advertising or product endorsement purposes.

This work performed under the auspices of the U.S. Department of Energy by Lawrence Livermore National Laboratory under Contract DE-AC52-07NA27344. 


\section{Instructions}

Click to

Continue

The Chemical Agent Incident-Response and Recovery Decision Process tool is an interactive document using a web browser to step through and link processes.

Each page is linked to following pages.

- To move forward, click on the arrow in the upper right-hand corner of each page.

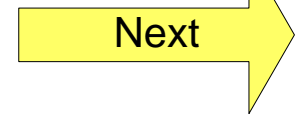

- To move backward, click on the arrow in the upper left corner.

\section{Previous}

Many of the process blocks have been expanded to show more detail. These blocks are indicated with an asterisk.

- Clicking on these blocks will open an expansion page. The block that is expanded will be duplicated in the upper right corner.

- To return to the original diagram, simply click on that block.

- As an alternative, there is an up-arrow button on the lower right corner which will also cause a return

$$
\hat{\imath}
$$




\section{Chemical Agent Incident-Response Decision Process (1 of 5)}
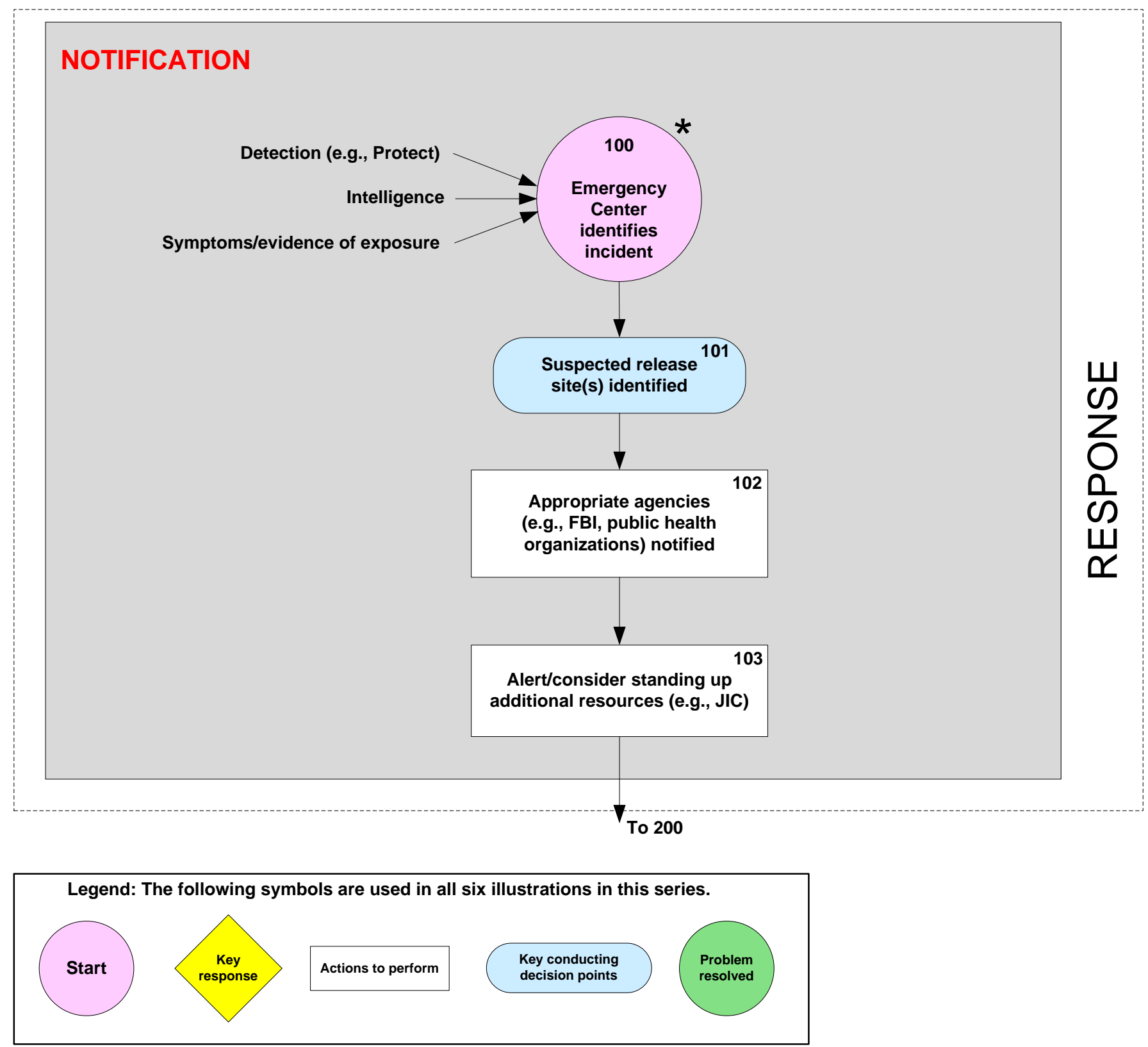

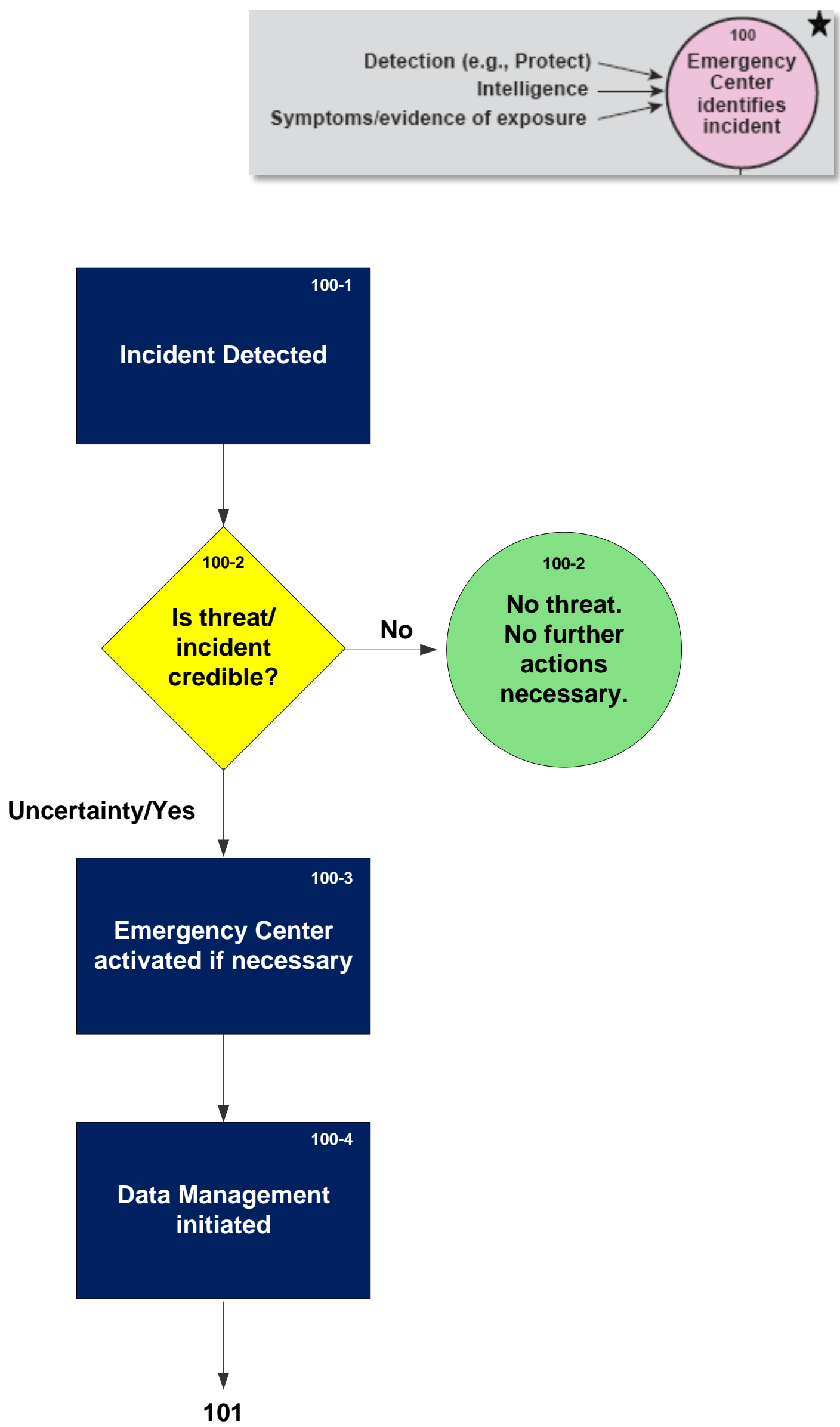


\section{Chemical Agent Incident-Response Decision Process (2 of 5)}

\section{FIRST RESPONSE}

From 103






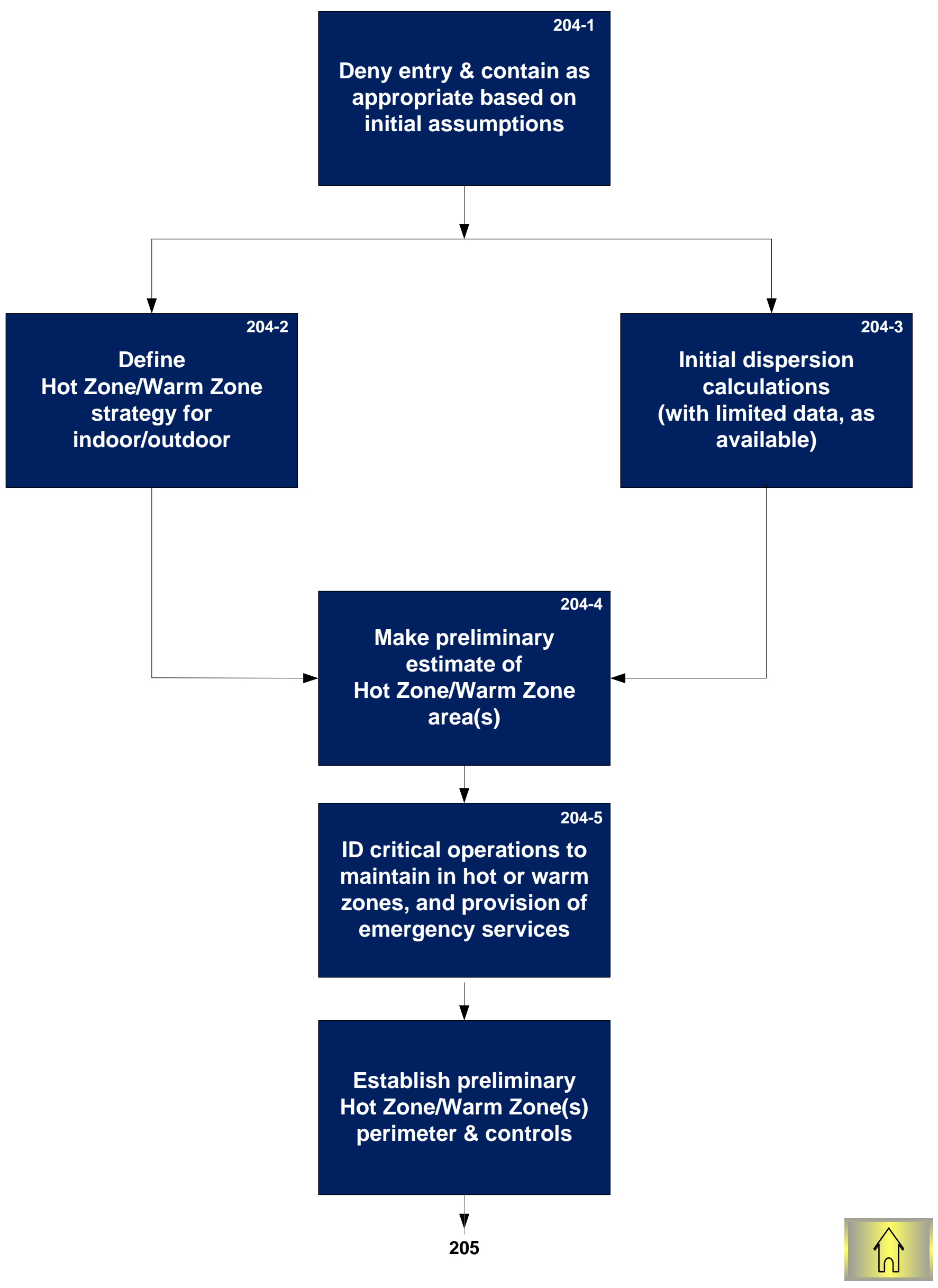




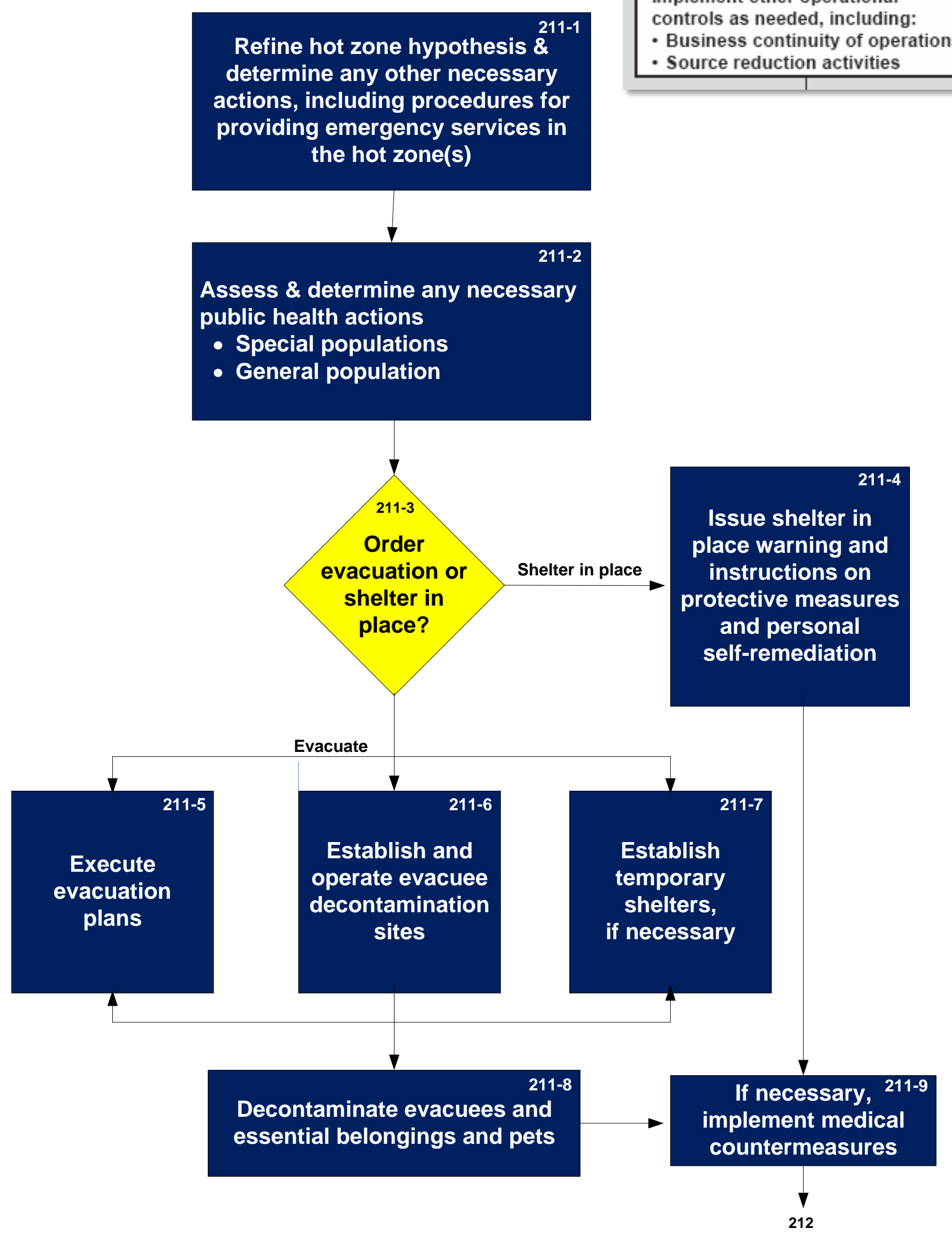

Use available agent-specific

information to perform any additional continuing HAZMAT and emergency-response actions (204-206).

Implement other operational controls as needed, including:

- Business continuity of operations

- Source reduction activities determine any other necessary actions, including procedures for

gemergency services in the hot zone(s)

\section{ublic health actions \\ - Special populations \\ - General population}

Assess \& determine any necessary 


\section{Chemical Agent Incident-Response Decision Process (3 of 5)}

\section{CHARACTERIZATION}

From 213

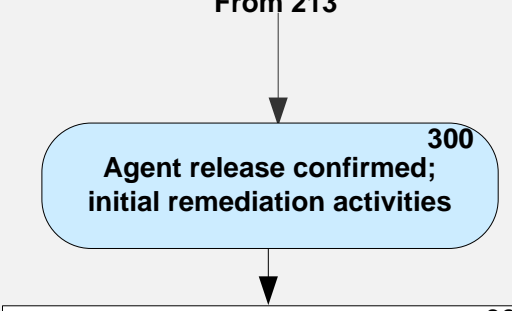

Identify and prioritize areas, operations, and/or facilities for detailed characterization/remediation

\section{$\nabla$}

Conduct detailed characterization for remediation purposes 302 (including information collected during First Response Phase)

- Time since release

- Concentration of agent

- Extent of contamination

- Estimation of exposure via all routes

- Characteristics of chemical agent

Is there potential impact to property/ environment?

Determine site-specific characteristics

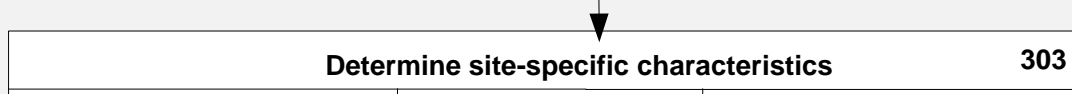

\begin{tabular}{|c|c|c|}
\hline \multicolumn{1}{|c|}{ Enclosed/Semi-enclosed } & Outdoor Areas & Water \\
\hline e.g., Size of facility & e.g., Meteorological & e.g., Potential for
\end{tabular}

ventilation systems, conditions, humidity, temperature, airflow, height of walls, specific building materials contamination of building intakes, soil type(s), surface run-off drinking water facilities and sources, $\mathrm{pH}$, redox potential, temperature, effects of dilution, flow rate

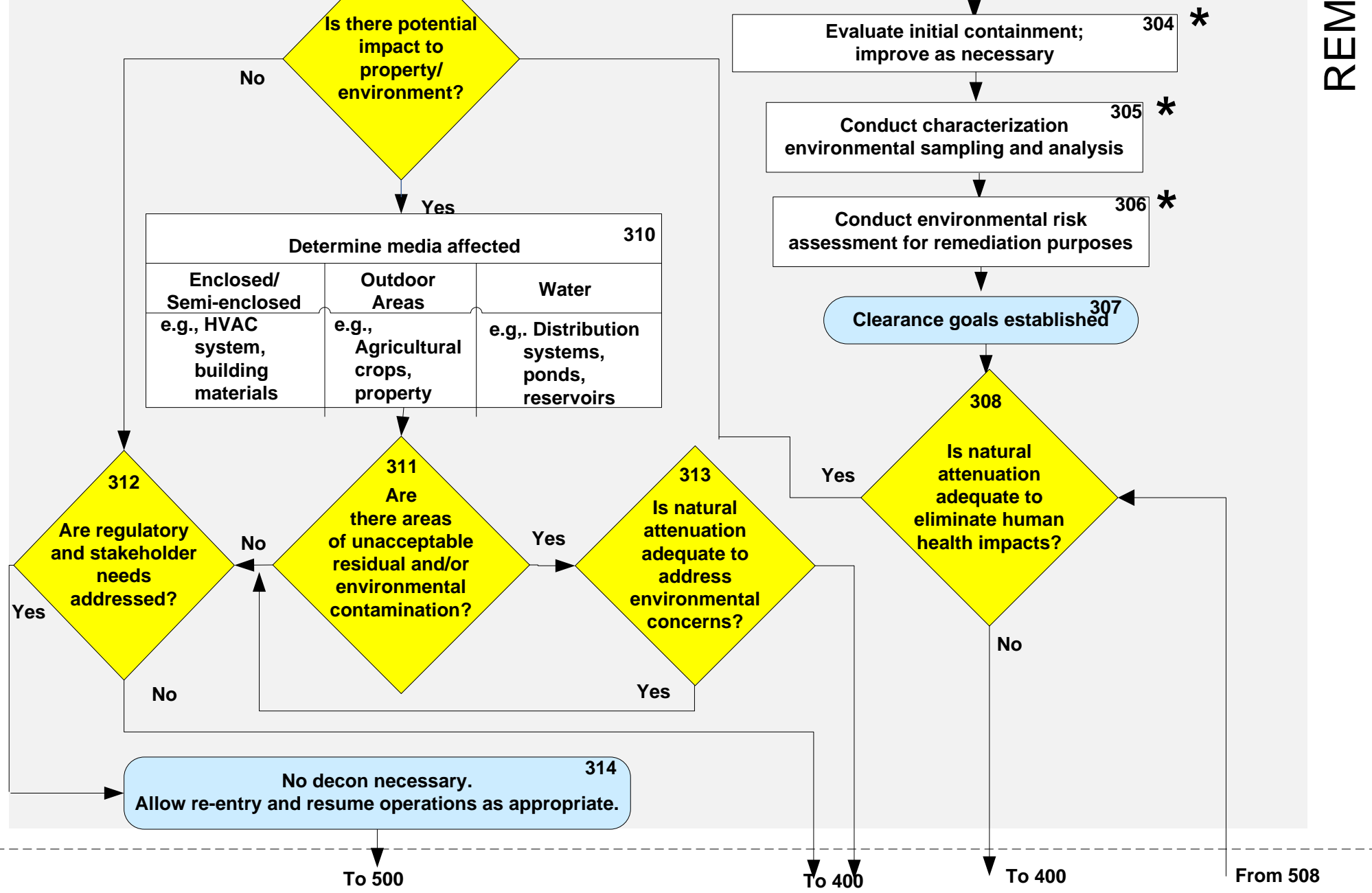


- Time since release

- Concentration of agent

- Extent of contamination

- Estimation of exposure via all routes

- Characteristics of chemical agent

\section{Gather/collect information from} first response (e.g., time since release, concentration maps, characteristics of agent)

$$
\nabla
$$

302-3

As task 303 progresses, continually reassess modeling results
Interpret and compare with contamination models verify models with response sample data

- Dispersion transport models (IMAAC)

- Indoor airflow models

- Water flow models

Identify data gaps necessitating additional characterization activities 


\begin{tabular}{|c|c|c|}
\hline \multicolumn{3}{|c|}{ Determine site specific characteristics } \\
\hline Enclosed/Semi-enclosed & Outdoor Areas & Water \\
\hline $\begin{array}{l}\text { e.g., Size of facility } \\
\text { ventilation systems, } \\
\text { humidity, temperature, } \\
\text { airflow, height of walls, } \\
\text { specific building materials }\end{array}$ & \begin{tabular}{|l} 
e.g., \\
coneteorological \\
building intakes, \\
soil type(s), \\
surface run-off
\end{tabular} & $\begin{array}{l}\text { e.g., Potential for contamination } \\
\text { of drinking water facilities } \\
\text { and sources, pH, redox } \\
\text { potential, temperature, } \\
\text { effects of dilution, flow rate }\end{array}$ \\
\hline
\end{tabular}

Determine contamination vulnerabilities and characteristics for all components within affected areas based on understanding of transport mechanisms

\begin{tabular}{|c|c|c|}
\hline $\begin{array}{c}\text { Enclosed/Semi-enclosed } \\
\text { Structures }\end{array}$ & Outdoor Areas & Water \\
\hline $\begin{array}{ll}\text { - } & \text { Exposure pathways } \\
\text { - } & \text { Facility transport systems } \\
\text { - } & \text { Porous/nonporous } \\
\text { surface areas } \\
\text { - } \quad \text { Characteristics of } \\
\text { materials } \\
\text { - } \\
\text { - } & \text { Poil presence } \\
\text { reservoirs }\end{array}$ & $\begin{array}{ll}\text { - } & \text { Soil types } \\
\text { - } & \text { Surfaces } \\
\text { - } & \text { Engetation } \\
\text { - } & \text { Tranditions } \\
\text { Transport } \\
\text { mechanisms } \\
\text { Vaporization or } \\
\text { reaerosolization } \\
\text { potential }\end{array}$ & $\begin{array}{l}\text { - All connections and components } \\
\text { of the drinking water system } \\
\text { All connections and components } \\
\text { of the surface water handling } \\
\text { system, to include runoff maps } \\
\text { and flow rates } \\
\text { Physico-chemical characteristics } \\
\text { of water in both drinking water } \\
\text { and runoff systems } \\
\text { All connections and components } \\
\text { of the waste water system }\end{array}$ \\
\hline
\end{tabular}

\section{Compile a list of potential} contamination sites

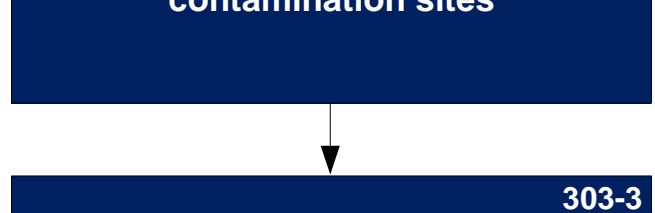

Map potential contamination sites/areas

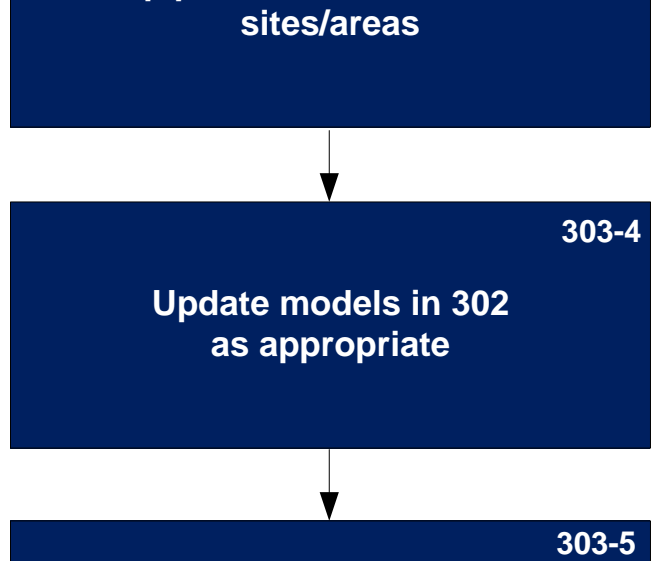

Develop characterization priorities, coordinating outdoor and enclosed/semi-enclosed areas 


\section{Evaluate initial containment;} improve as necessary

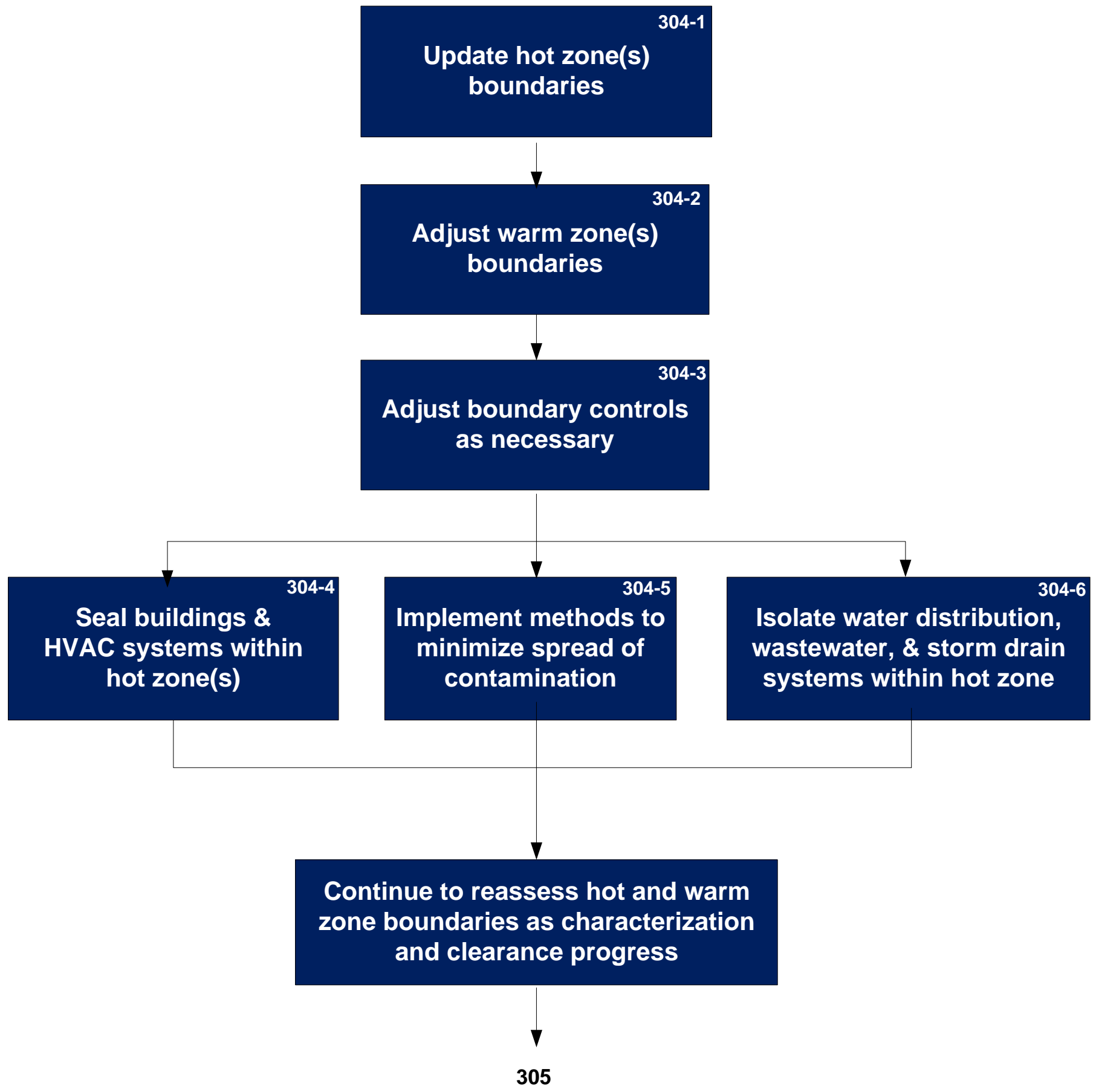


Conduct characterization environmental sampling and analysis

Develop comprehensive characterization sampling strategies

- Consider a phased approach to rapidly refine the limits of contamination

- Develop data quality objectives

- Consider statistical and judgmental criteria

- Employ iterative modeling approach to optimize sampling

- Establish minimum characterization sampling requirements

- Consider employing a zonal approach for sampling

- Consider surface and air sampling

- Address all necessary information requirements for risk assessment (including inhalation, ocular, dermal, and ingestion risks)
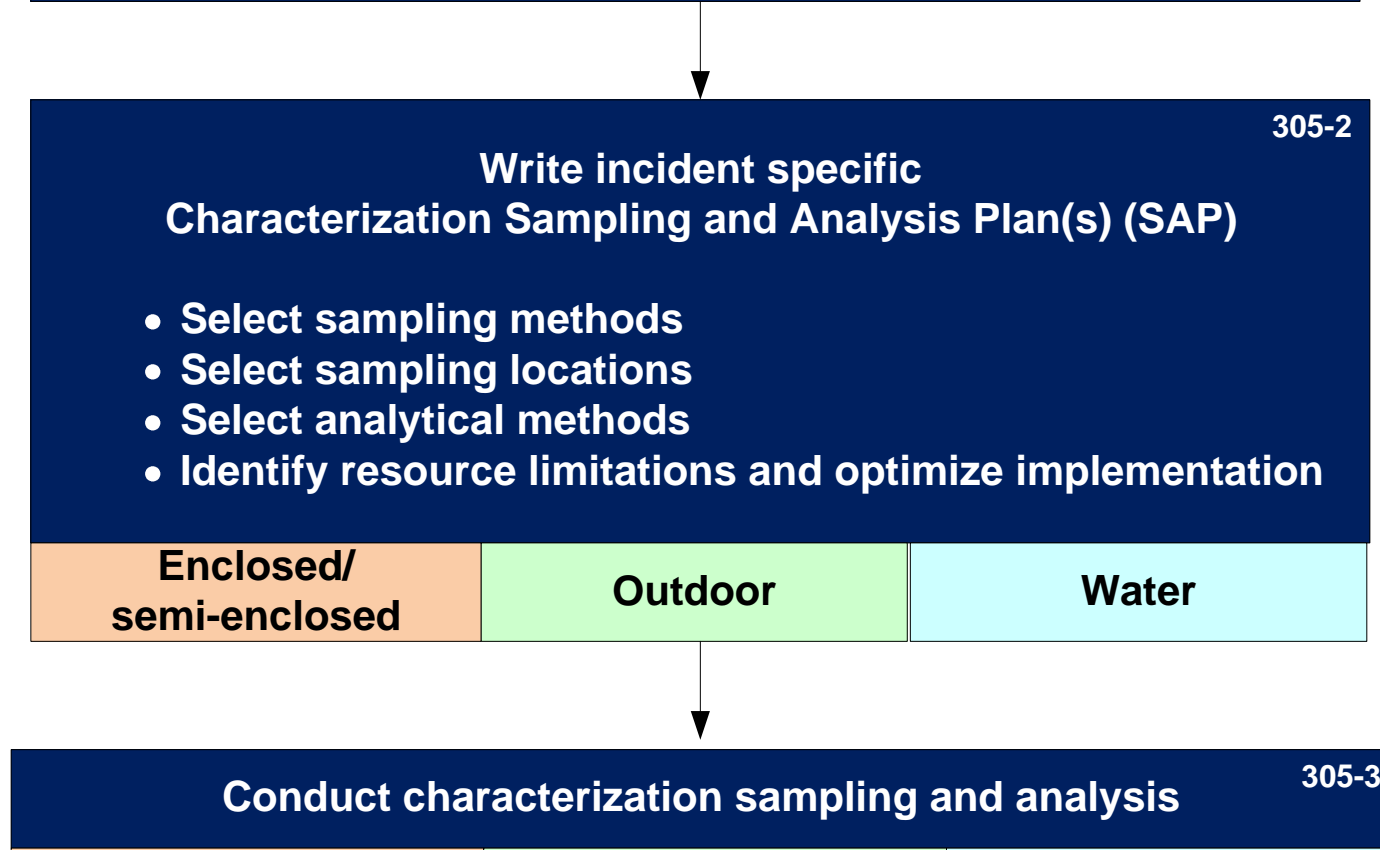

\begin{tabular}{|c|c|c|}
\hline $\begin{array}{c}\text { Enclosed/ } \\
\text { semi-enclosed }\end{array}$ & Outdoor & Water \\
\hline
\end{tabular}

Re-evaluate and adjust sampling strategy, plans, and methods as necessary 

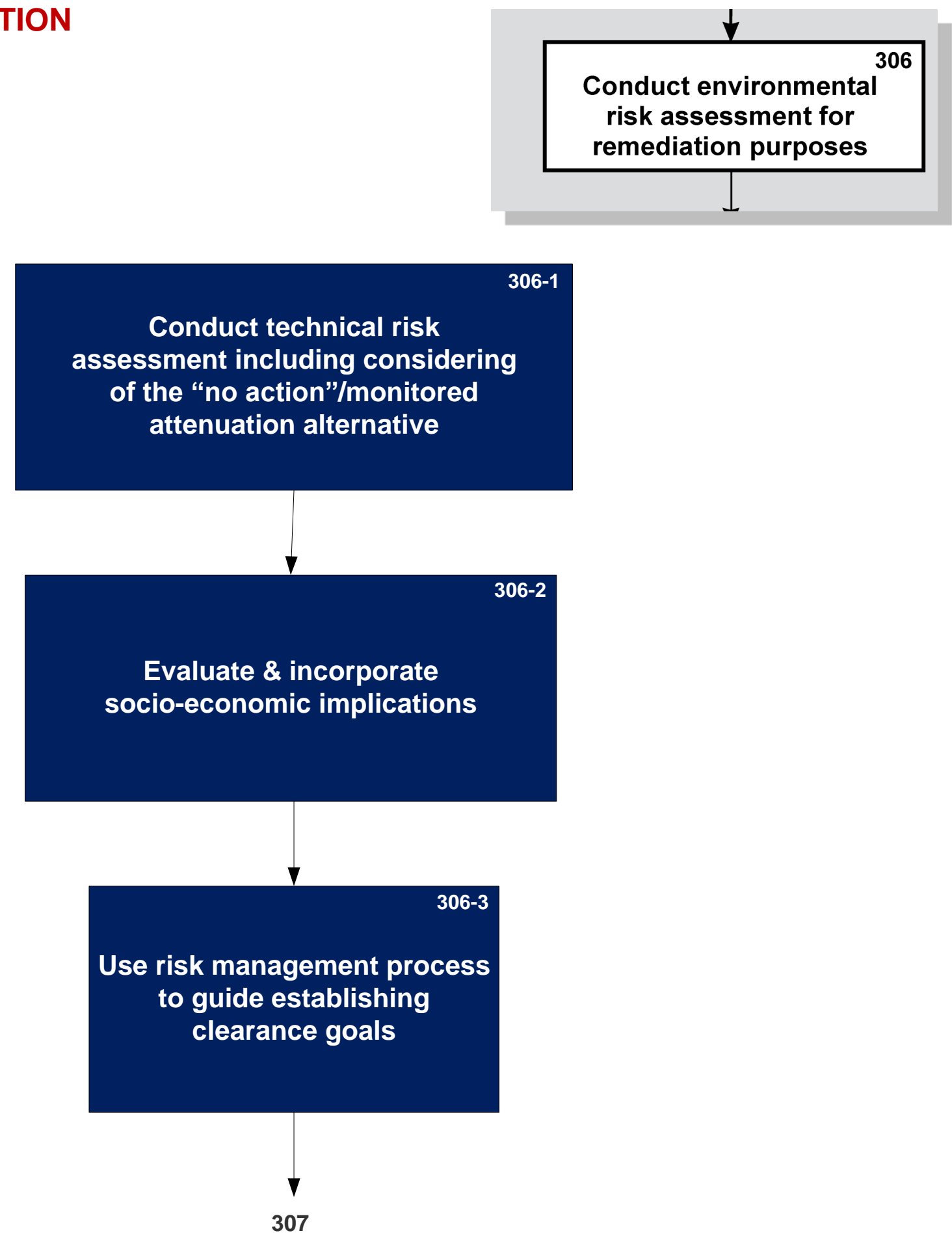


\section{Chemical Agent Incident-Response Decision process (4 of 5)}

\section{DECONTAMINATION}

Removal and Disposal

\section{Determine} decontamination options (employ multiple options as appropriate)



Select appropriate treatment and * disposal site; determine packaging and transportation requirements

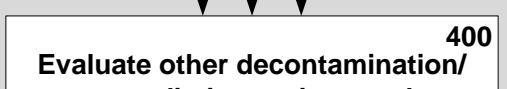

remediation options and necessary regulatory requirements No (Recycle/Reuse)



- Address clearance goals

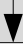

Conduct decontamination and 406 remediation actions as needed

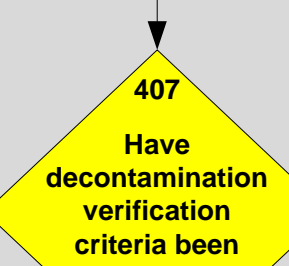

criteria been

met?

Yes (in situ) 


\begin{tabular}{|c|l|l|l|}
\hline \multicolumn{4}{|c|}{ Evaluate decontamination options for media affected, } \\
considering for example: \\
Volume, Extent of contamination, Availability of resources, Accessibility, Weather \\
\hline \multicolumn{1}{|c|}{$\begin{array}{c}\text { Enclosed/ } \\
\text { Semi-enclosed }\end{array}$} & \multicolumn{2}{|c|}{ Outdoor Areas } & \multicolumn{1}{|c|}{ Water } \\
\hline $\begin{array}{l}\text { e.g., HVAC system, } \\
\text { building materials, fixed \& } \\
\text { moveable property, } \\
\text { sensitive equipment, } \\
\text { rolling stock }\end{array}$ & $\begin{array}{l}\text { Natural } \\
\text { e.g., soil, } \\
\text { vegetation, } \\
\text { agricultural } \\
\text { crops, } \\
\text { livestock }\end{array}$ & $\begin{array}{l}\text { Man-made } \\
\text { e.g., building } \\
\text { exteriors, } \\
\text { pavement } \\
\text { structures, } \\
\text { fixed and } \\
\text { movable } \\
\text { property, } \\
\text { sensitive } \\
\text { equipment, } \\
\text { rolling stock }\end{array}$ & $\begin{array}{l}\text { e.g., Distribution } \\
\text { systems, ponds, } \\
\text { reservoirs, } \\
\text { recreational water, } \\
\text { surface run-off }\end{array}$ \\
\hline & & & \\
\hline
\end{tabular}

Identify and asses consistency

of decon options with

regulatory requirements 
Select decontamination methods including specific reagents and reagent delivery systems for media affected, considering for example:

Effectiveness, Volume, Extent of contamination, Availability of resources, Accessibility, Weather

\begin{tabular}{|c|c|c|c|}
\hline $\begin{array}{c}\text { Enclosed/ } \\
\text { Semi-enclosed }\end{array}$ & \multicolumn{2}{|c|}{ Outdoor Areas } & Water \\
\hline \multirow{2}{*}{$\begin{array}{l}\text { e.g., HVAC system, } \\
\text { building materials, } \\
\text { fixed and moveable } \\
\text { property, sensitive } \\
\text { equipment } \\
\text { rolling stock } \\
\text { Considering: } \\
\text { - Surface treatment } \\
\text { - Volumetric methods }\end{array}$} & $\begin{array}{l}\text { Natural } \\
\text { e.g., soil, } \\
\text { vegetation, } \\
\text { agricultural, } \\
\text { crops, livestock }\end{array}$ & $\begin{array}{l}\text { Man-made } \\
\text { e.g., building } \\
\text { exteriors, } \\
\text { pavement } \\
\text { structures, fixed } \\
\text { and moveable } \\
\text { property, sensitive } \\
\text { equipment, rolling } \\
\text { stock }\end{array}$ & \multirow{2}{*}{$\begin{array}{l}\text { e.g., distribution } \\
\text { systems, ponds, } \\
\text { reservoirs, recreational } \\
\text { water, surface run-off } \\
\\
\text { Considering: } \\
\text { - Treatment } \\
\text { - No treatment and } \\
\text { monitor }\end{array}$} \\
\hline & \multicolumn{2}{|c|}{$\begin{array}{l}\text { Considering: } \\
\text { - Localized treatment } \\
\text { - Wide area treatment } \\
\text { - Wash down and treat } \\
\text { - } \quad \text { Monitored natural attenuation }\end{array}$} & \\
\hline
\end{tabular}

Develop emergency response plans to address potential uncontrolled reagent release(s)

$\nabla$

Develop worker safety strategies for each method

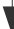

Identify stakeholder concerns for each decon method selected

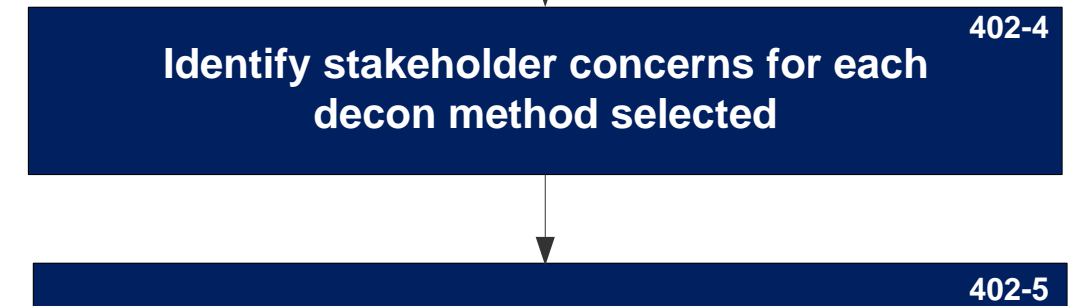

Document comprehensive decontamination plans

for incorporation in the RAP including prioritization of decontamination activities 
Develop appropriate decontamination strategy; determine packaging and transportation requirements

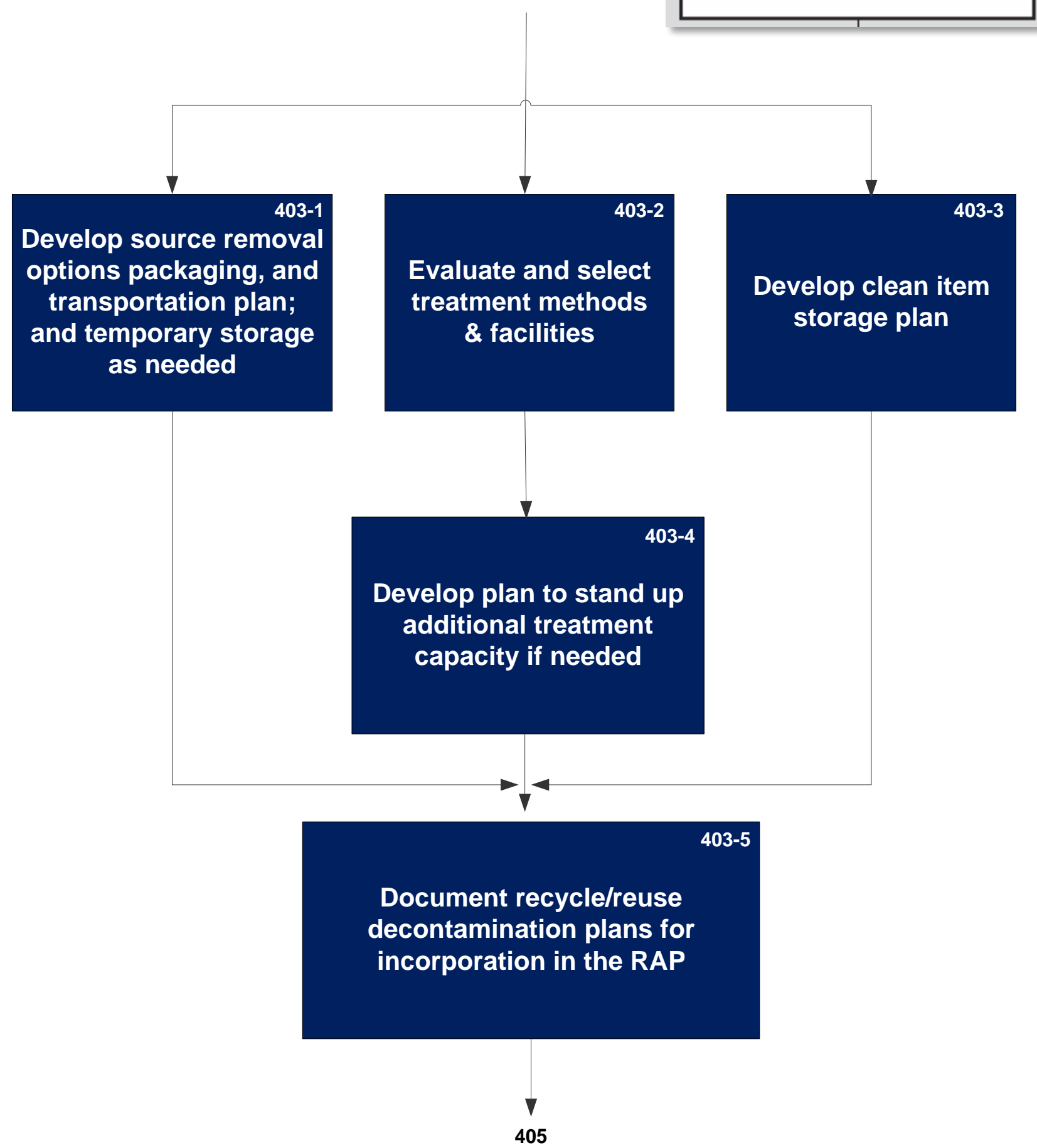




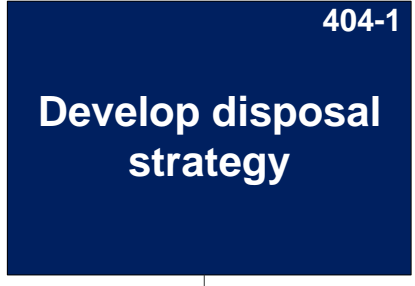

$\nabla$

Select removal, packaging, and transportation methods; and temporary storage solutions as needed

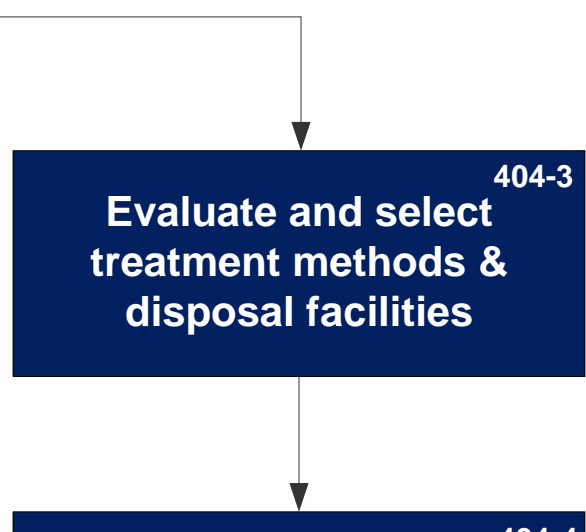

Develop plan to stand up additional treatment $\&$ disposal capacity if needed

Document disposal plans for

incorporation in the RAP 


\section{Update prioritization of areas for decontamination}

\section{5-1}

\section{$\nabla$}

Develop integrated decontamination strategy, considering;

- Effectiveness

- Priorities and optimization of resources

- Decontamination of outdoor areas before enclosed/semi-enclosed areas

Develop decon verification criteria and process criteria (e.g. concentration, temperature, humidity, contact time, pH)

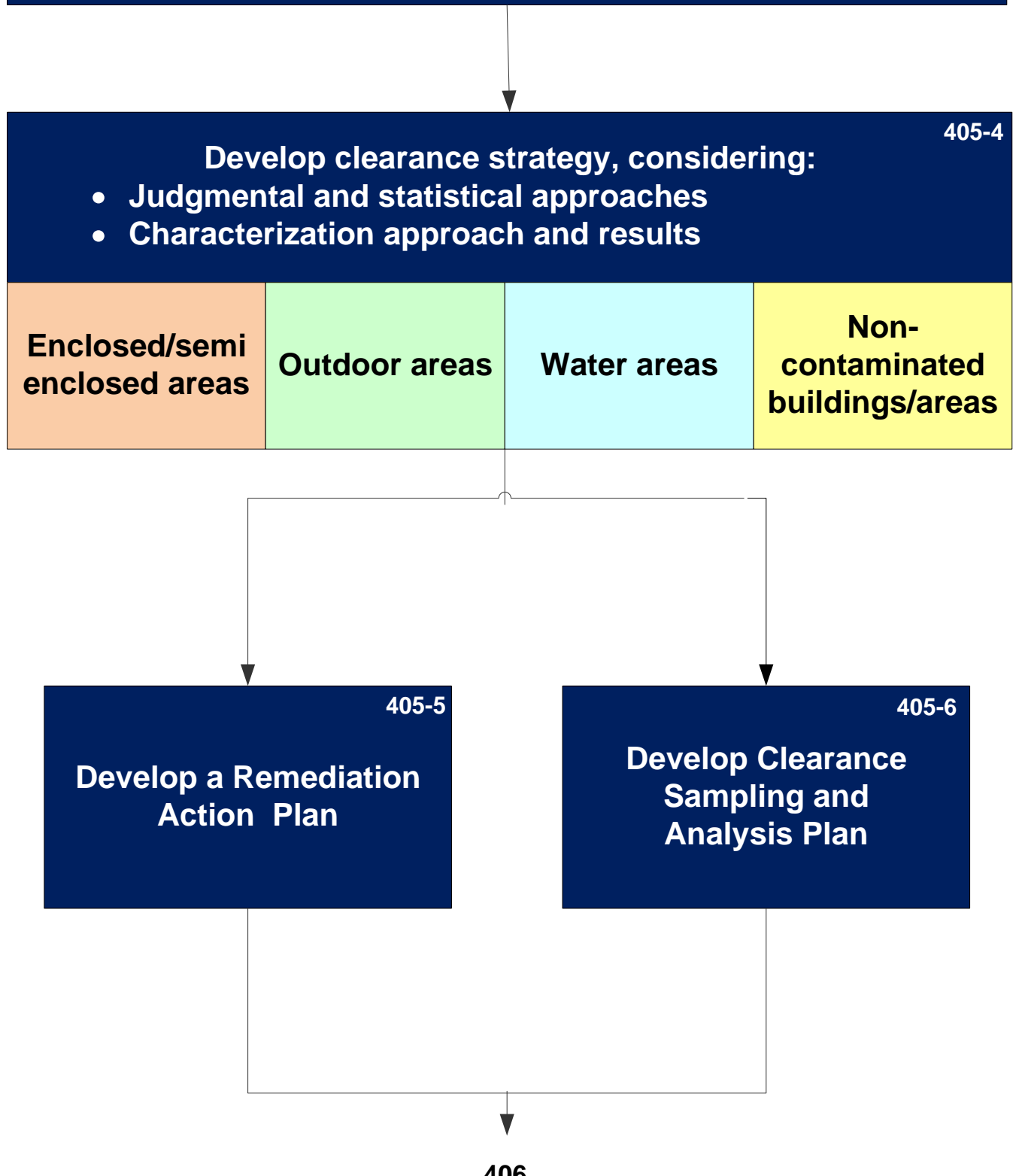




\section{Previous}

Chemical Agent Incident-Response Decision Process (5 of 5)

From 314

From 408

To 400

To 307

To 400

1

$\Lambda$

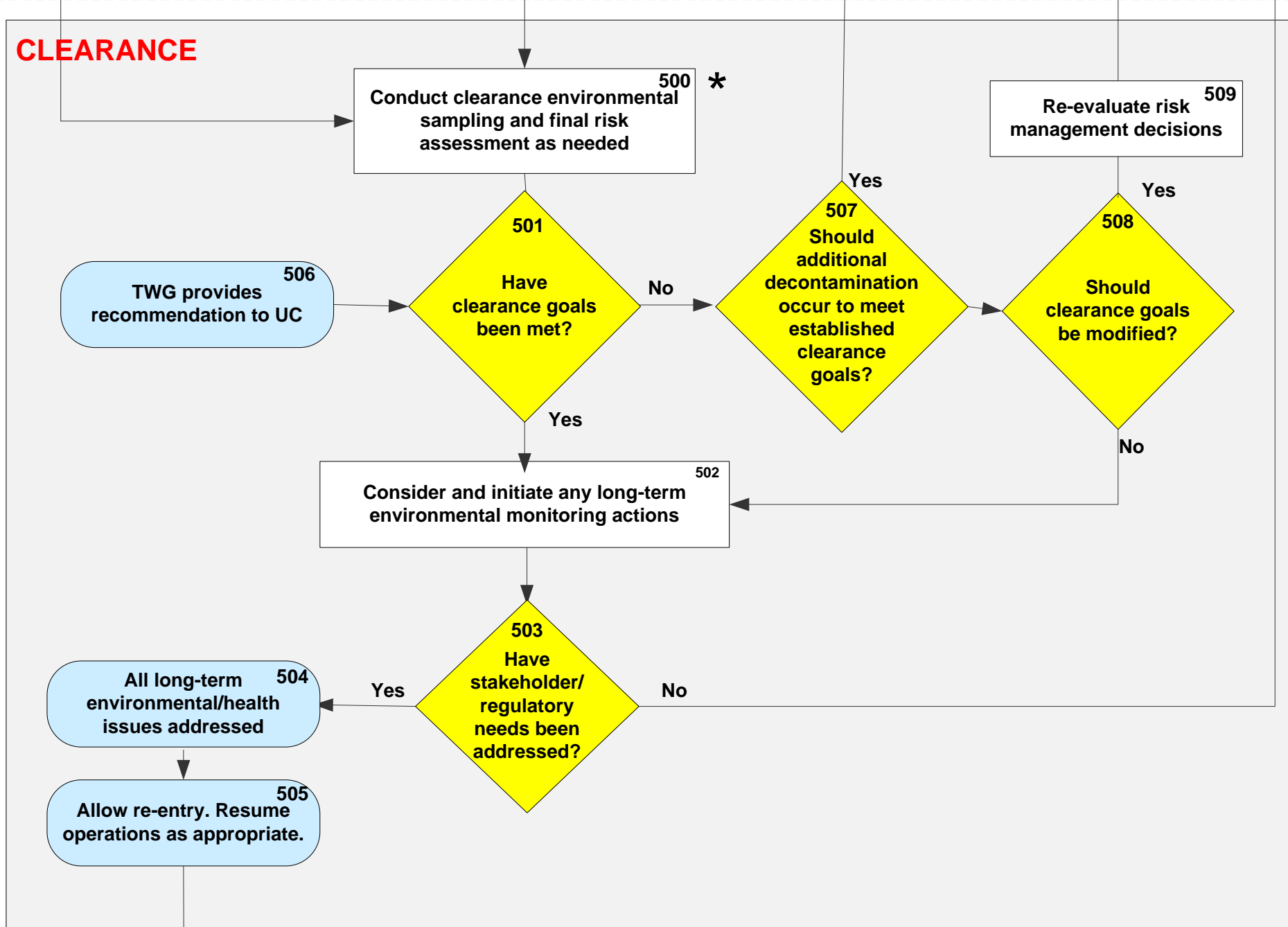

\section{REOCCUPANCY}
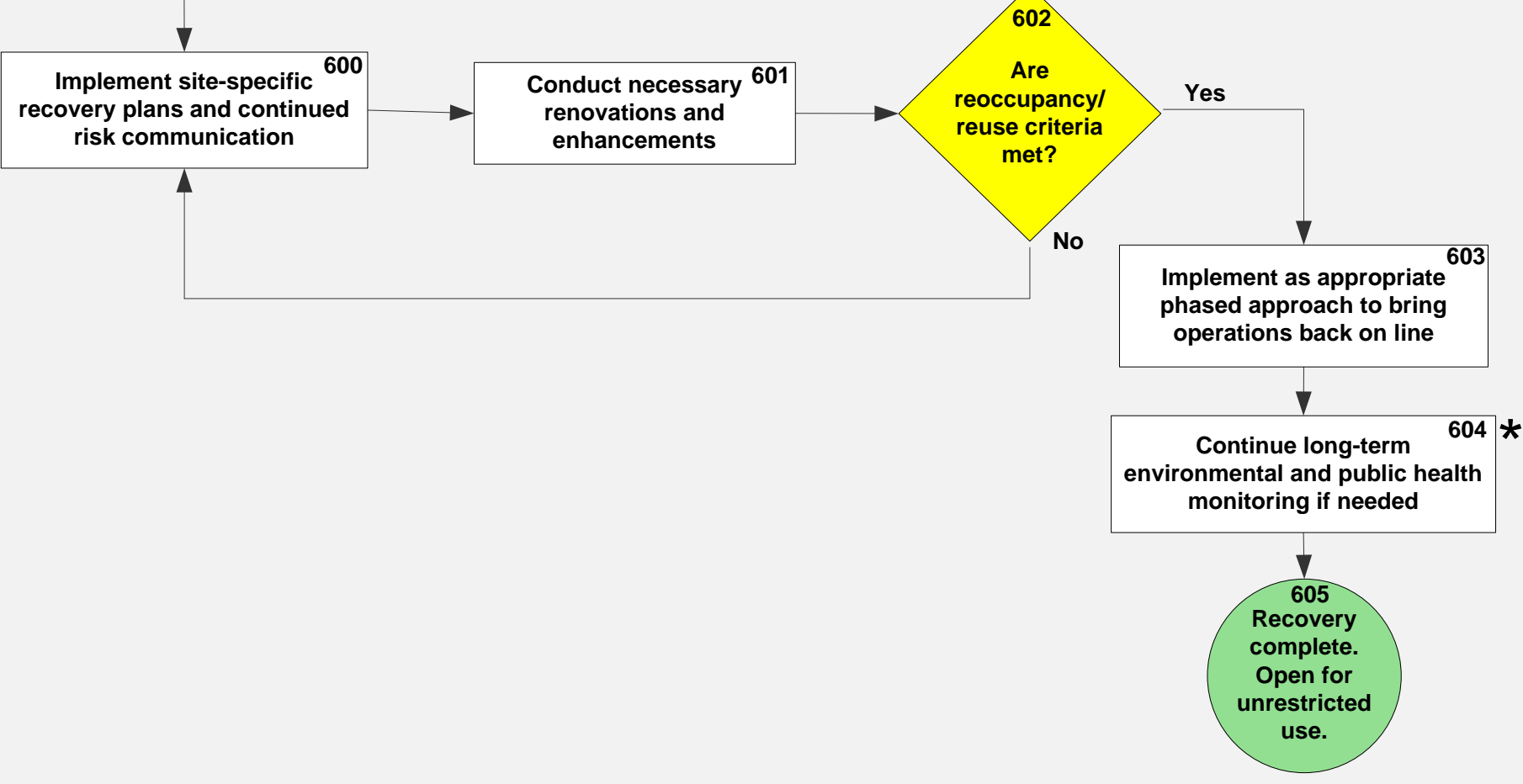
CLEARANCE

(expanded 500)

\section{Conduct clearance $\quad 500$ \\ environmental sampling and final risk assessment as needed}

Reassess need for clearance

in non-contaminated

potentially impacted areas

$500-2$

Reassess Clearance Sampling and Analysis Plan based on priorities, dependencies, and potential for recontamination

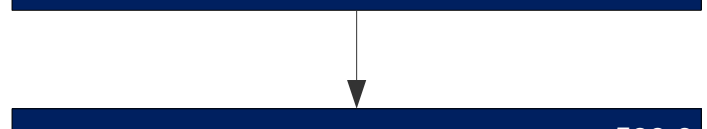

Execute Clearance Sampling and Analysis Plan

Conduct final technical risk assessment, considering results from clearance sampling

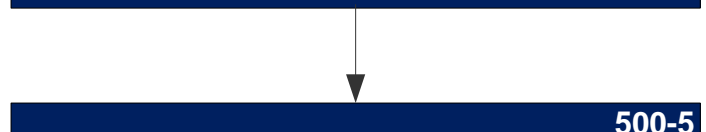

500-5

Consider socio-economic implications 
Continue long-term environmental and public health monitoring if needed

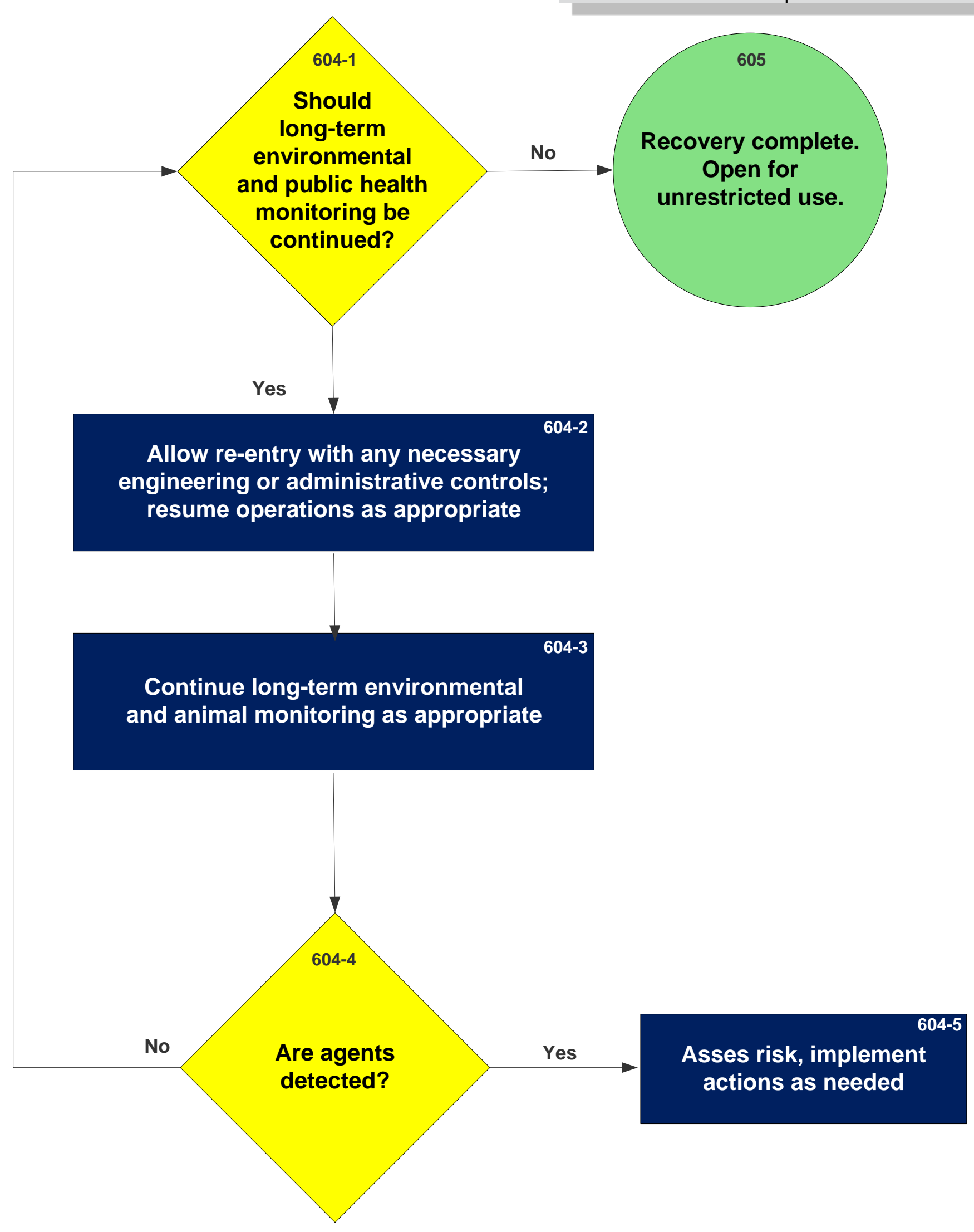

